\title{
Multi-purpose HealthCare Telemedicine Systems with mobile communication link support
}

\author{
E Kyriacou ${ }^{* 1,2}$, S Pavlopoulos ${ }^{1}$, A Berler ${ }^{1}$, M Neophytou $^{1}$, A Bourka1, \\ A Georgoulas ${ }^{1}$, A Anagnostaki1, D Karayiannis ${ }^{3}$, C Schizas², C Pattichis ${ }^{2}$, \\ A Andreou ${ }^{2}$ and D Koutsouris ${ }^{1}$
}

\author{
Address: ${ }^{1}$ Biomedical Engineering Laboratory, Department of Electrical and Computer Engineering, National Technical University of Athens \\ (NTUA), Athens, Greece, ${ }^{2}$ Department of Computer Science, University of Cyprus (UCY), Nicosia, Cyprus and ${ }^{3}$ Athens Medical Center (AMC), \\ Athens, Greece \\ Email: E Kyriacou* - ekyriac@ucy.ac.cy; S Pavlopoulos - spav@biomed.ntua.gr; A Berler - berler@biomed.ntua.gr; \\ M Neophytou - mneoph@ucy.ac.cy; A Bourka - abourka@biomed.ntua.gr; A Georgoulas - ageorg@biomed.ntua.gr; \\ A Anagnostaki - angiea@biomed.ntua.gr; D Karayiannis - dikar@iatriko.gr; C Schizas - schizas@ucy.ac.cy; C Pattichis - pattichi@ucy.ac.cy; \\ A Andreou - aandreou@ucy.ac.cy; D Koutsouris - dkoutsou@biomed.ntua.gr \\ * Corresponding author
}

Published: 24 March 2003

BioMedical Engineering OnLine 2003, 2:7
Received: 30 November 2002

Accepted: 24 March 2003

This article is available from: http://www.biomedical-engineering-online.com/content/2/1/7

(c) 2003 Kyriacou et al; licensee BioMed Central Ltd. This is an Open Access article: verbatim copying and redistribution of this article are permitted in all media for any purpose, provided this notice is preserved along with the article's original URL.

\begin{abstract}
The provision of effective emergency telemedicine and home monitoring solutions are the major fields of interest discussed in this study. Ambulances, Rural Health Centers (RHC) or other remote health location such as Ships navigating in wide seas are common examples of possible emergency sites, while critical care telemetry and telemedicine home follow-ups are important issues of telemonitoring. In order to support the above different growing application fields we created a combined real-time and store and forward facility that consists of a base unit and a telemedicine (mobile) unit. This integrated system: can be used when handling emergency cases in ambulances, $\mathrm{RHC}$ or ships by using a mobile telemedicine unit at the emergency site and a base unit at the hospital-expert's site, enhances intensive health care provision by giving a mobile base unit to the ICU doctor while the telemedicine unit remains at the ICU patient site and enables home telemonitoring, by installing the telemedicine unit at the patient's home while the base unit remains at the physician's office or hospital. The system allows the transmission of vital biosignals (3-12 lead ECG, SPO2, NIBP, IBP, Temp) and still images of the patient. The transmission is performed through GSM mobile telecommunication network, through satellite links (where GSM is not available) or through Plain Old Telephony Systems (POTS) where available. Using this device a specialist doctor can telematically "move" to the patient's site and instruct unspecialized personnel when handling an emergency or telemonitoring case. Due to the need of storing and archiving of all data interchanged during the telemedicine sessions, we have equipped the consultation site with a multimedia database able to store and manage the data collected by the system. The performance of the system has been technically tested over several telecommunication means; in addition the system has been clinically validated in three different countries using a standardized medical protocol.
\end{abstract}




\section{Background}

Telemedicine is defined as the delivery of health care and sharing of medical knowledge over a distance using telecommunication means. Thus, the aim of Telemedicine is to provide expert-based health care to understaffed remote sites and to provide advanced emergency care through modern telecommunication and information technologies. The concept of Telemedicine was introduced about 30 years ago through the use of nowadayscommon technologies like telephone and facsimile machines. Today, Telemedicine systems are supported by State of the Art Technologies like Interactive video, high resolution monitors, high speed computer networks and switching systems, and telecommunications superhighways including fiber optics, satellites and cellular telephony [1].

The availability of prompt and expert medical care can meaningfully improve health care services at understaffed rural or remote areas. The provision of effective emergency Telemedicine and home monitoring solutions are the major fields of interest discussed in this study. There are a wide variety of examples where those fields are crucial. Nevertheless, Ambulances, Rural Health Centers (RHC) and Ships navigating in wide seas are common examples of possible emergency sites, while critical care telemetry and Telemedicine home follow-ups are important issues of telemonitoring. In emergency cases where immediate medical treatment is the issue, recent studies conclude that early and specialized pre-hospital patient management contributes to the patient's survival [2]. Especially in cases of serious head injuries, spinal cord or internal organs trauma, the way the incidents are treated and transported is crucial for the future well being of the patients.

A quick look to past car accident statistics points out clearly the issue: During 1997, 6753500 incidents were reported in the United States [3] from which about 42000 people lost their lives, 2182660 drivers and 1125890 passengers were injured. In Europe during the same period 50000 people died resulting of car crash injuries and about half a million were severely injured. Furthermore, studies completed in 1997 in Greece [4], a country with the world's third highest death rate due to car crashes, show that $77,4 \%$ of the 2500 fatal injuries in accidents were injured far away from any competent healthcare institution, thus resulting in long response times. In addition, the same studies reported that $66 \%$ of deceased people passed away during the first 24 hours.

Coronary artery diseases is another common example of high death rates in emergency or home monitoring cases since still two thirds of all patients die before reaching the central hospital. In a study performed in the UK in 1998 [5], it is sobering to see that among patient above 55 years old, who die from cardiac arrest, $91 \%$ do so outside hospital, due to a lack of immediate treatment. In cases where thrombolysis is required, survival is related to the "call to needle" time, which should be less than 60 minutes [6]. Thus, time is the enemy in the acute treatment of heart attack or sudden cardiac death (SCD). Many studies worldwide have proven that a rapid response time in prehospital settings resulting from treatment of acute cardiac events decreases mortality and improves patient outcomes dramatically [7]-[12]. In addition, other studies have shown that 12-lead ECG performed during transportation increase available time to perform thrombolytic therapy effectively, thus preventing death and maintaining heart muscle function [13]. The reduction of all those high death rates is definitely achievable through strategies and measures, which improve access to care, administration of pre-hospital care and patient monitoring techniques.

Critical care telemetry is another case of handling emergency situations. The main point is to monitor continuously intensive care units' (ICU) patients at a hospital and at the same time to display all telemetry information to the competent doctors anywhere, anytime [14]. In this pattern, the responsible doctor can be informed about the patient's condition at a 24-hour basis and provide vital consulting even if he's not physically present. This is feasible through advanced telecommunications means or in other words via Telemedicine.

Another important Telemedicine application field is home monitoring. Recent studies show that [15] the number of patients being managed at home is increasing, in an effort to cut part of the high hospitalization's cost, while trying to increase patient's comfort. Using low-cost televideo equipment that runs over regular phone lines, providers are expanding the level while reducing the frequency of visits to healthcare institutions [16]. In addition, a variety of diagnostic devices can be attached to the system giving to the physician the ability to see and interact directly with the patient. For example, pulse oximetry and respiratory flow data can be electronically transmitted (for patients with chronic obstructive pulmonary disease). Diabetes patients can have their blood glucose and insulin syringe monitored prior to injection for correct insulin dosage. Furthermore, obstetric patients can have their blood pressure and fetal heart pulses monitored remotely and stay at home rather than prematurely admitted to a hospital.

It is common knowledge that people that monitor patients at home or are the first to handle emergency situations do not always have the required advanced theoretical background and experience to manage properly all cases. Emergency Telemedicine and home 
monitoring can solve this problem by enabling experienced neurosurgeons, cardiologists, orthopedics and other skilled people to be virtually present in the emergency medical site. This is done through wireless transmission of vital biosignals and on scene images of the patient to the experienced doctor. A survey [17] of the Telemedicine market states that emergency Telemedicine is the fourth most needed Telemedicine topic with $39.8 \%$ coverage of market requests while home healthcare covers $23.1 \%$. The same survey also points out that the use of such state of the art technologies has $23 \%$ enhanced patient outcomes.

Several systems that could cover emergency cases [18][23], home monitoring cases [24]-[25] and critical care telemetry [14] have been presented over the years. Recent developments in mobile telecommunications and information technology enhanced capability in development of telemedicine systems using wireless communication means [26]-[32]. In most cases however only the store and forward procedure was successfully elaborated, while the great majority of emergency cases do require real time transmition of data.

In order to cover as much as possible of the above different growing demands we created a combined real-time and store and forward facility that consists of a base unit and a telemedicine unit where this integrated system:

- Can be used when handling emergency cases in ambulances, RHC or ships by using the Telemedicine unit at the emergency site and the expert's medical consulting at the base unit

- Enhances intensive health care provision by giving the telemedicine unit to the ICU doctor while the base unit is incorporated with the ICU's in-house telemetry system

- Enables home telemonitoring, by installing the telemedicine unit at the patient's home while the base unit remains at the physician's office or hospital.

The Telemedicine device is compliant with some of the main vital signs monitor manufacturers like Johnson \& Johnson CRITIKON Dinamap Plus and Welch Allyn Protocol (Propaq). It is able to transmit both 3 and 12 lead ECGs, vital signs (non-invasive blood pressure, temperature, heart rate, oxygen saturation and invasive blood pressure) and still images of a patient by using a great variety of communication means (Satellite, GSM and Plain Old Telephony System - POTS). The base unit is comprised of a set of user-friendly software modules that can receive data from the Telemedicine device, transmit information back to it and store all data in a database at the base unit. The communication between the two parts is based on the TCP/IP protocol. The general framework for the above system was developed under EU funded TAP (Telematics Applications Programme) projects, the EMERGENCY 112 project(HC 4027)[33] and the Ambulance project(HC1001) [22].

\section{Methods}

\section{Trends and needs of Telemedicine systems}

As mentioned above, scope of this study was to design and implement an integrated Telemedicine system, able to handle different Telemedicine needs especially in the fields of:

- Emergency health care provision in ambulances, Rural Hospital Centers (or any other remote located health center) and navigating Ships

\section{- Intensive care patients monitoring}

- Home telecare, especially for patients suffering from chronic and /or permanent diseases (like heart disease).

In other words we determined a "Multi-purpose" system consisting of two major parts: a) Telemedicine unit (which can be portable or not portable depending on the case) and b) Base unit or doctor's unit (which can be portable or not portable depending on the case and usually located at a Central Hospital).

Figure 1 describes the overall system architecture. In each different application the Telemedicine unit is located at the patient's site, whereas the base unit (or doctor's unit) is located at the place where the signals and images of the patient are sent and monitored. The Telemedicine device is responsible to collect data (biosignals and images) from the patient and automatically transmit them to the base unit. The base unit is comprised of a set of user-friendly software modules, which can receive data from the Telemedicine device, transmit information back to it and store important data in a local database. The system has several different applications (with small changes each time), according to the current healthcare provision nature and needs.

Before the system's technical implementation, an overview of the current trends and needs in the aforementioned Telemedicine applications was made, so that the different requirements are taken into account during design and development, thus ensuring maximum applicability and usability of the final system in distinct environments and situations. Table 1 provides the results of this overview, which was done towards a predefined list of criteria that usually influence a Telemedicine application implementation (cost, portability, autonomy, weight and size of Telemedicine device, type and quality of PC and camera, communication means used). 


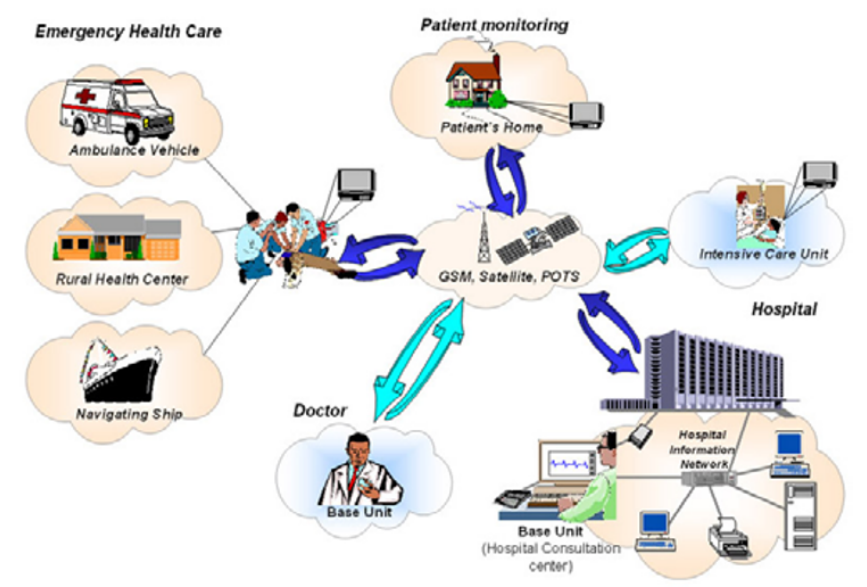

Figure I

Overall system architecture

As shown in the Table 1, low cost is a very crucial aspect for home telecare, since the costs are covered by the patient and not by the hospital (in contrast with all other applications). Portability, autonomy and small weight \& size of the Telemedicine device are a very important component in ambulance applications, where the device also needs to be transferred at the scene (outside the ambulance). This is also related with the PC type, which in ambulances must be a palmtop or a sub notebook, while in other cases can be desktop or laptop. The camera quality in all applications should be as high as possible, but in certain cases like the intensive care room it is of up most importance. As far as communication is considered, in ambulances and ships, GSM is the major mean, while in RHC and homecare it is POTS. Satellite links are suggested mainly for ships, but it should always be taken into account that costs arise very much with the quality of the links (in other words, a lot of money should be spent to obtain reliable equipment for transmission via satellite links). User friendliness is important in all applications, but even more important in home telecare, where not specialized or trained staff is using the device.

Besides the above, the Telemedicine applications can be examined towards other criteria, like for example security needs, transmission type (continuos, store \& forward) needs, ECG leads required (3 or 12 leads), etc. These last are examined in more detail in the next paragraph, where the overall technical description of the system is provided.

\section{System design and technical implementation}

As mentioned above, the system consists of two separate modules (Figure 1): a) the unit located at the patient's site called "Telemedicine unit" and b) the unit located at doc-

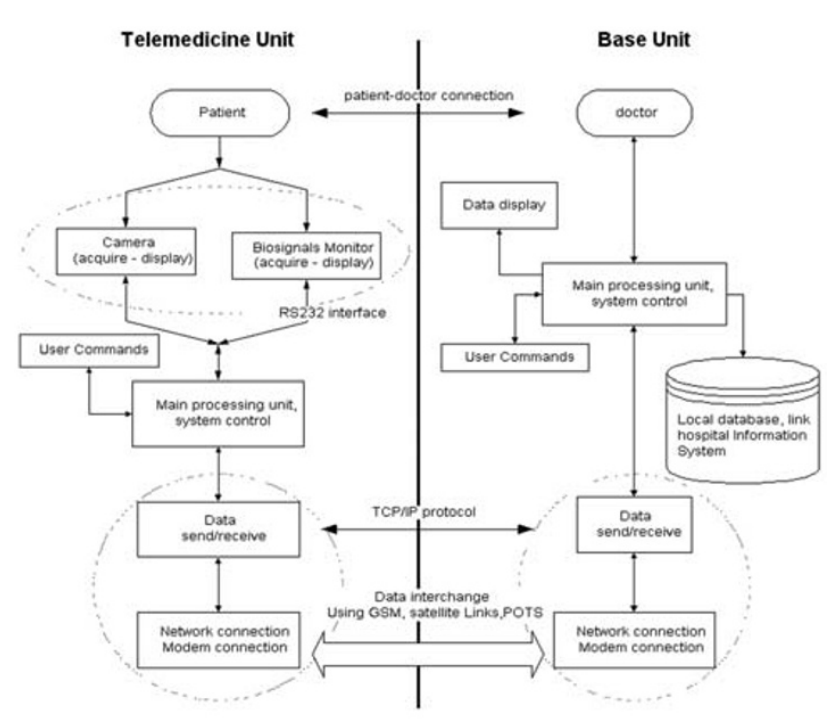

Figure 2

Information Flow within the Telemedicine system (Telemedicine and base units)

tor's site called "Base Unit". The Doctor might be using the system either in an Emergency case or when monitoring a patient from a remote place.

The design and implementation of the system was based on a detailed user requirements analysis, as well as the corresponding system functional specifications. The study was mainly based on the experience of Telemedicine projects named AMBULANCE [22] and Emergency 112 [33] where functional prototypes of a device with emergency Telemedicine functionalities was built and extensively evaluated. Through these project we had phased the need to implement a telemedicine device, which would facilitate a flexible architecture and could be used in several emergency or monitoring cases that have simiral needs of information transmition.

The Telemedicine unit is responsible for collecting and transmitting biosignals and still images of the patients from the incident place to the Doctor's location while the Doctor's unit is responsible for receiving and displaying incoming data. The information flow (using a layered description) between the two sites can be seen in Figure 2.

The software design and implementation follows the client server model; it was done using Borland Delphi 5 [34] for windows 95/98/NT/2000 platform; the Telemedicine unit site is the client while the Base unit site is the server. Communication between the two parts is achieved using TCP/IP as network protocol, which ensures safe data 
Table I: Overview of current trends and needs in Telemedicine applications

\begin{tabular}{|c|c|c|c|c|c|c|c|c|}
\hline \multirow{2}{*}{$\begin{array}{l}\text { Telemedicine } \\
\text { applications }\end{array}$} & \multicolumn{8}{|l|}{ Basic needs } \\
\hline & Cost & Portability & Autonomy & $\begin{array}{l}\text { Small } \\
\text { Weight } \\
\text { \& size }\end{array}$ & PC type & $\begin{array}{l}\text { Camera } \\
\text { quality }\end{array}$ & $\begin{array}{l}\text { Communi- } \\
\text { cation } \\
\text { means }\end{array}$ & $\begin{array}{l}\text { User } \\
\text { friendliness }\end{array}$ \\
\hline Ambulance & Medium/High & High & High & High & Palmtop & Medium High & GSM & Medium/High \\
\hline RHC & Medium/High & Low & Low & Low & Desktop Laptop & Medium High & POTS, GSM & Medium/High \\
\hline Ship & Medium/High & Low/ Medium & Low/ Medium & Low & Desktop Laptop & Medium High & GSM, Satellite & Medium/High \\
\hline Home care & Low & Low/ Medium & Low/ Medium & Low & Desktop Laptop & High & POTS & High \\
\hline $\begin{array}{l}\text { Intensive care } \\
\text { room }\end{array}$ & Medium/High & Low & Low & Low & Desktop & High & POTS, GSM & Medium/High \\
\hline
\end{tabular}

transmission and interoperability over different telecommunication means (GSM, Satellite, and POTS). System communications are based on a predefined communication protocol for data interchange, which is used to control and maintain connection between the two sites, thus ensuring portability, interoperability and security of the transmitted data. During the design and implementation phase an extended codification scheme based on the "Vital" and DICOM" was developed [35]. Based in this experience we had created the communication protocol.

\section{a) Telemedicine Unit}

The Telemedicine unit mainly consists of four modules, the biosignal acquisition module, which is responsible for biosignals acquisition, a digital camera responsible for image capturing, a processing unit, which is basically a Personal Computer, and a communication module (GSM, Satellite or POTS modem).

The biosignal acquisition module was designed to operate with some of the most common portable biosignal monitors used in emergency cases or in Intensive care Units such as a) CRITIKON DINAMAP PLUS Monitor Model 8700/9700 family of monitors, b) PROTOCOL-Welch Allyn Propaq 1xx Vital Signs Monitor, c) PROTOCOL-Welch Allyn Propaq Encore 2xx Vital Signs Monitor.

The biosignals collected by the patient (and then transmitted to the Base Unit) are:

- ECG up to 12 lead, depending on the monitor used in each case.

- Oxygen Saturation (SpO2).

- Heart Rate (HR).

- Non-Invasive Blood Pressure (NIBP).
- Invasive blood Pressure (IP).

- Temperature (Temp)

- Respiration (Resp)

The PC used depends on the type of the Telemedicine application (role of the Telemedicine unit). As shown in Table 1: a) in cases where the autonomy and small size of the system are important (mainly in ambulances), a sub notebook like Toshiba libretto $100 \mathrm{ct}$ portable PC is used, a picture of a portable device is shown in Figure $3 \mathrm{~b}$ ) in cases where we need some autonomy but size is not considered an important element a Typical Pentium portable PC is used; c) in cases where we do not necessarily need

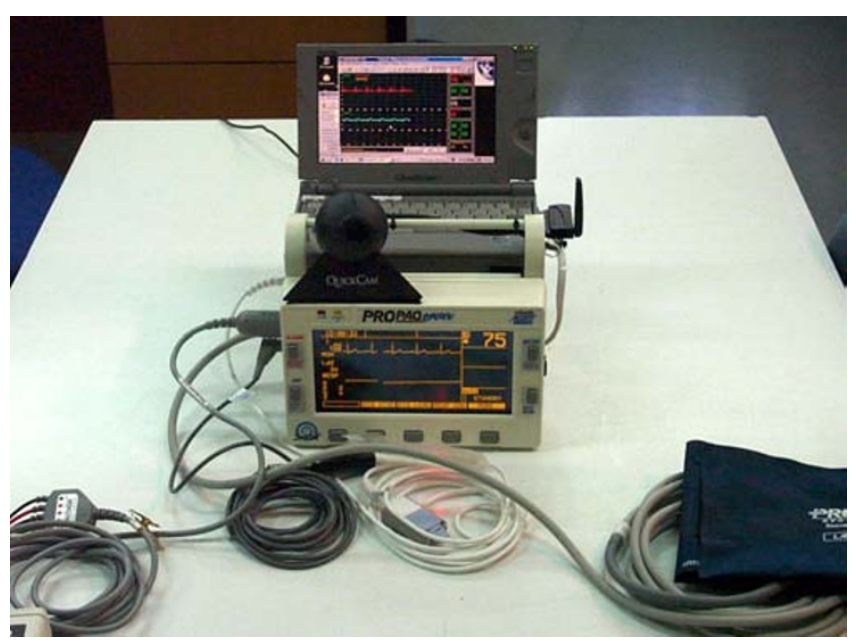

Figure 3

Picture of telemedicine mobile unit (monitor Propaq $2 x x$ is used) 
autonomy, portability and small system size, a Typical Pentium Desktop PC is used.

As mentioned before, data interchange is done using the TCP/IP network protocol, which allows operation over several communication means. The PC is equipped with the proper modem for each case, i.e. GSM, Satellite or POTS. The design was done for standard Hayes modems. The system supports ETSI - AT command set for GSM modem, for Satellite modems and for Standard POTS modems. Several modems types were used for testing: a) a NOKIA card phone 2.0 GSM 900/1800 modem pcmcia card and an Option FirstFone GSM 900 modem pcmcia card were used for GSM communication, b) a Micronet pcmcia POTS modem $56 \mathrm{~K}$ and a US-Robotics $33.6 \mathrm{~K}$ external modem were user for POTS communication, c) a mini m terminal for ships "Thrane \& Thrane TT-3064A CAPSAT Inmarsat Maritime Phone" was used for satellite communication.

The Telemedicine unit is also responsible for the collection and transmission of images of the patient to the base unit. In order to implement a hardware independent system, this module was designed to operate using Microsoft video for WINDOWS. Several cameras were used while testing the system: a) ZOOM digital camera connected to the PC's parallel port model 1585. b) ZOOM digital camera connected to the PC's usb port model 1595. c) Logitech quick cam express digital usb port camera d) Connectix quick cam VC parallel port e) Creative camera connected to usb port.

The control of the Telemedicine unit is fully automatic. The only thing the telemedicine unit user has to do is connect the biosignal monitor to the patient and turn on the PC. The PC then performs the connection to the base unit automatically. Although the base unit basically controls the overall system operation, the Telemedicine unit user can also execute a number of commands. This option is useful when the system is used in a distance health center or in a ship and a conversation between the two sites takes place.

\section{b) Base Unit (or Doctor's Unit)}

The base unit mainly consists of a dedicated PC equipped with a modem, which is responsible for data interchange. In addition the base unit pc is responsible for displaying incoming signals from the Telemedicine unit. When an expert doctor uses the base unit located outside the hospital area (like in the Intensive Care Room application - see Figure 1, a portable PC equipped with a GSM modem or a desktop PC equipped with a POTS modem is used. When the base unit is located in the hospital, a desktop PC connected to the Hospital Information Network (HIS) equipped with a POTS modem can additionally be used; the expert doctor uses it as a processing terminal.

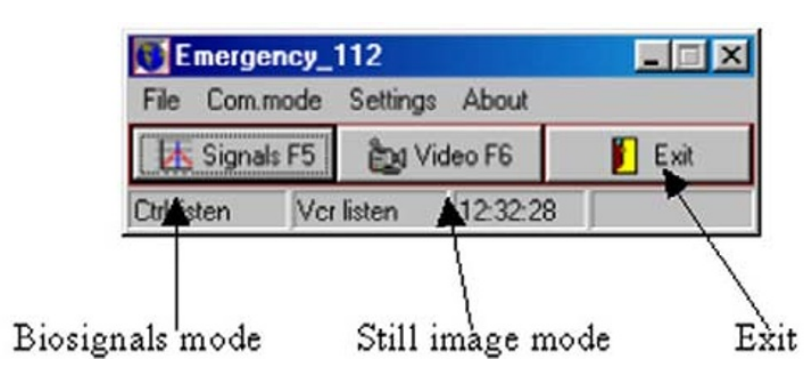

\section{Figure 4}

Control Window - Base Unit

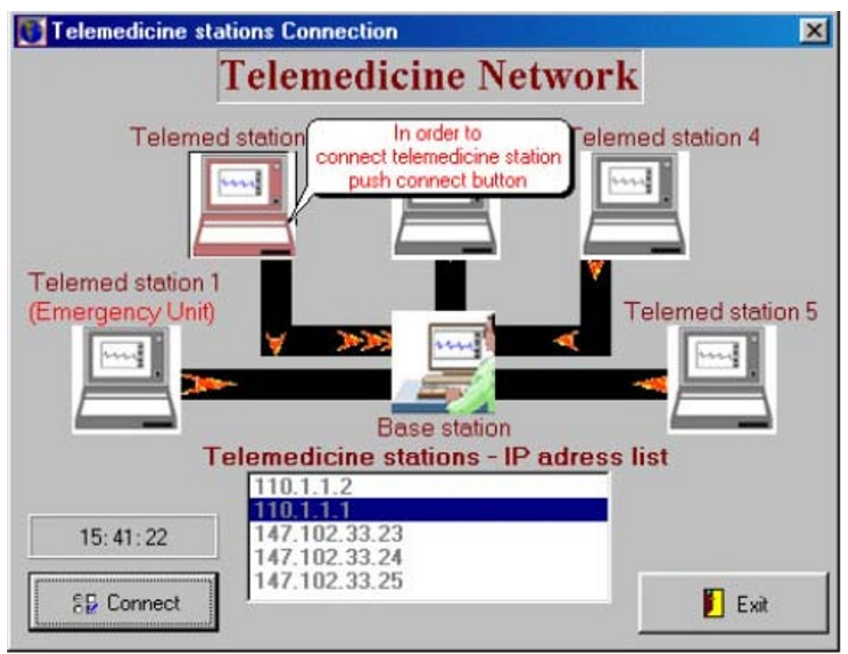

Figure 5

Telemedicine Network control - Base Unit

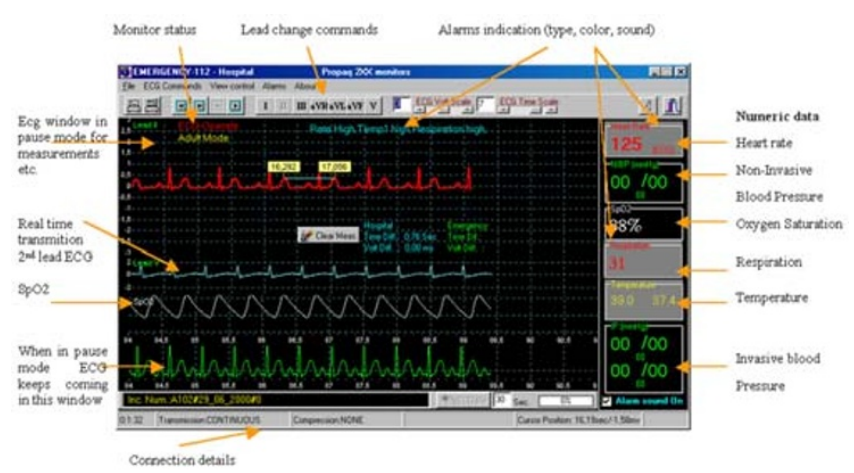

Figure 6

Biosignal receiving window at Base Unit 
Through the base unit, user has the full control of the telemedicine session. The user is able to monitor the connection with a client (telemedicine unit), send commands to the telemedicine unit such as the operation mode (biosignals or images) Figure 4. In cases were the base station is connected to a Hospital LAN the user can choose to which of the telemedicine units to connect to, as shown in Figure 5 the user of the base unit is able to choose and connect to anyone of the telemedicine units connected on the network. The units connected on the network can be ICU telemedicine units or distance mobile telemedicine units connected through phone lines.

The Base Unit's user can monitor biosignals or still images coming from the Telemedicine unit, thus keeping a continuous online communication with the patient site. This unit has the full control of the Telemedicine session. The doctor (user) can send all possible commands concerning both still image transmission and biosignals transmission. Figure 6 presents a typical biosignal-receiving window (continuous operation).

When the system operates on still image mode, the doctor can draw-annotate on the image and send the annotations back to the Telemedicine unit. The Telemedicine Unit user can also annotate on the freezed image and annotations will then again be transferred to the Base unit.

When operating on biosignal mode (Figure 6), the transmission of vital biosignals can be done in two ways, continuous way or store and forward way, depending on the ECG waveform channels which are transmitted and the telecommunication channel data transfer rate. In continuous operation, the Base Unit user can send commands to the Telemedicine Unit monitor, such as lead change or blood pressure determination; the user can also pause incoming ECG, move it forward or backward and perform some measurements on the waveform.

\section{b) Hospital database Unit}

When the Base Unit is located in a hospital (especially in emergency handling or in home telecare), a Hospital database unit can be integrated in the system, in order to record information concerning the cases handled. When the system is used for emergency cases, predefined information for each case are registered, information includes incident's number, date, time, initial and final diagnosis, Telemedicine files etc. This information is compliant to the directive "Standard Guide for View of Emergency Medical Care in the Computerized Patient Record" (designation E1744-95) of the American Society for Testing and Materials (ASTM). When the system is used in an Ambulance Emergency Medical Service, the database unit is also responsible for accepting and recording emergency calls, as well as managing Ambulance vehicle fleet.

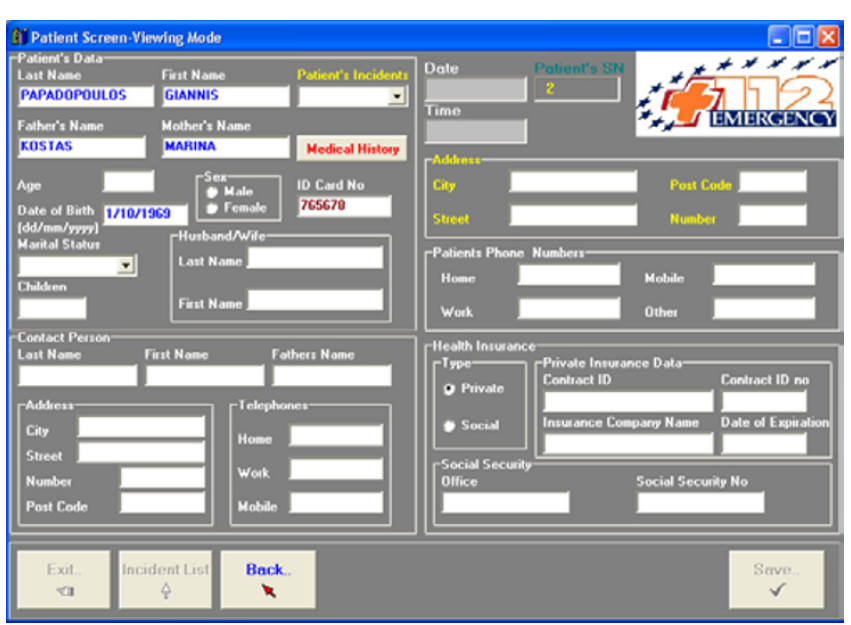

Figure 7

Patient Information window, Hospital database Unit

In cases where a Hospital Information System (HIS) is already available at the Base Unit site (Hospital), the doctor (Base Unit user) can retrieve information (using the hospital archiving unit) concerning the patient's medical history. When HIS is not available, the Hospital Database Unit can handle the patient medical record by itself (Figure 7).

The database was designed using Paradox 7 and was equipped with graphical user interface features built in Borland Delphi 5 for increased user friendliness. All parts of the database are in compatibility with Microsoft Windows 95/98/NT/2000. For security reasons, according to the directive 95/46/EC, the database is fully protected against unauthorized access and is password protected and encrypted, whereas the whole application is password protected with several access levels depending on user groups.

\section{c) Technical Constraints - Feasibility \\ Biosignals transmission}

Along with biosignals, information concerning the monitor, such as the alarms or the monitor status, is transmitted from the Telemedicine unit to the base unit. The ECG waveform and $\mathrm{SpO} 2$ or $\mathrm{Co} 2$ Waveform (where available) is the continuous signals transmitted, trends are transmitted for the rest of data. ECG data are sampled at a rate of $200 \mathrm{samples} / \mathrm{sec}$ by $10 \mathrm{bits} / \mathrm{sample}$ or $12 \mathrm{bits} / \mathrm{sample}$, for all monitors used, thus resulting in a generation of 2000 bits/sec and 2400 bits/sec for one ECG channel. SpO2 and Co2 waveforms are sampled at a rate of 100 samples/sec by $10 \mathrm{bit} / \mathrm{sample}$; thus resulting in a generation of 1000 bits/sec for one channel. Trends for SpO2, HR, NIBP, BP, 
Table 2: ECG channels and way of transferring over several Telecommunication means

\begin{tabular}{lllll}
\hline ECG signal Tel. Mean & I ECG Channel & 2 ECG Channel & $\begin{array}{l}\text { 2 ECG Channel +Other } \\
\text { waveform (Spo2 Co2) }\end{array}$ & I2 ECG Channel \\
\hline \multirow{2}{*}{ GSM } & $\begin{array}{l}\text { Continuous / Store \& } \\
\text { Forward }\end{array}$ & $\begin{array}{l}\text { Continuous / Store \& } \\
\text { Forward }\end{array}$ & $\begin{array}{l}\text { Continuous / Store \& } \\
\text { Forward }\end{array}$ & Store \& Forward \\
POTS & $\begin{array}{l}\text { Continuous / Store } \\
\text { \&Forward }\end{array}$ & $\begin{array}{l}\text { Forward } \\
\text { Stontinuous / Store \& }\end{array}$ & Store \& Forward \\
Satellite & Store \& Forward & Forward \& Forward & Store \& Forward & Store \&Forward \\
\hline
\end{tabular}

Temp and monitor data are updated with a refresh rate of one per second, thus adding a small fraction of data to be transmitted approximately up to $200 \mathrm{bits} / \mathrm{sec}$. All biosignals monitors used with the system can provide digital output of the collected signals [36-38].

\section{Image transmission}

Images captured by the Telemedicine unit's camera have resolution $320 \times 240$ pixel and are compressed using the JPEG compression algorithm; the resulting data set is approximately 5-6 KB depending on the compression rate used for the JPEG algorithm [39].

\section{Transmission rate}

The signals transmission is done using GSM, Satellite and POTS links. For the time being, the GSM network that the system was technically tested on; allows transmission of data up to 9600 bps (when operating on the normal mode) and is able to reach up to 43200 bps when using the HSDC (High Speed Circuit Switched Data). The satellite links transmission rate depends on the equipment and the satellite system used in each case; it has a range from $2400 \mathrm{bps}$ up to 64000 bps. The use of different satellite systems can increase the cost of equipment and cost of use; in our case we had used an INMARSAT-phone Mini$\mathrm{m}$ system which can transmit data only up to $2400 \mathrm{bps}$, but has low equipment and use cost. Plain Old Telephony System (POTS) allows the transmission of data using a rate up to $56000 \mathrm{bps}$, thus enabling the continuous and fast information transmission (Table 2).

The practical maximum data transfer rate over telecommunication means is never as high as the theoretical data transfer rate. Practical data rates depend on the time and the area where the system is used. Biosignals data transmission can be done in two ways: real time transmission where a continuous signal is transmitted from client to server or store and forward transmission where signals of a predefined period of time are stored in the client and transmitted as files to server. It mainly depends on the maximum data transfer rate of the telecommunication link used and the digital data output that the biosignal monitor has in each case.

\section{Waveforms Transmission}

As mentioned above monitors from two of the major portable monitors firms were used in this study, which can provide three to twelve leads waveform of ECG and numeric data from other biosignals (HR, SpO2, NIBP, IP, Temp).

The first of the monitors used, CRITIKON DINAMAP PLUS Monitor has a digital output of a continuous one channel ECG plus biosignals such as NIBP, SpO2, HR, IP and data concerning monitor alarms etc.; all the above information can be transferred using up to 2200 bps. For this reason, the continuous transmission of signals from this monitor can be done when using GSM and POTS and 2400 BPS satellite links.

The second of the monitors used, PROTOCOL Propaq Monitor has a digital output of a continuous one (model $1 \mathrm{xx}$ ) or two (model 2xx) channels ECG, plus another waveform such as $\mathrm{SpO} 2$ or $\mathrm{Co} 2$; plus biosignals trends such as NIBP, SpO2, HR, IP and data concerning monitor alarms etc. All above information can be transferred using up to 2400 BPS for one channel ECG, up to 4400 for two channels of ECG or up to 5400 for two channels of ECG plus another waveform ( $\mathrm{SpO} 2$ or $\mathrm{Co} 2)$. For this reason, the continuous transmission of signals from this monitor can be done when using GSM and POTS but only one lead ECG when using 2400 BPS satellite links.

\section{Compression \& encryption}

In order to decrease data size, a lossless ECG compression algorithm based on Huffman coding algorithm [40] is implemented in the system and can be applied on transmitted signals, when needed by the Base Unit user.

Security of the Telemedicine Unit was designed according to the directive $97 / 66 / \mathrm{EC}$, concerning processing of medical data in the telecommunication sector. An encryption algorithm was implemented in the system and can be used when needed by the hospital unit user. The system can encrypt interchanged data using the Blowfish cipher algorithm [41]. The use of encryption is optional and can be selected by the user; authentication and connection 
between base and telemedicine units is done using encrypted messages. In any case, in the communication between the Telemedicine and the base unit only the incident's ID number is used, while the patient's name or any other relevant information are never mentioned, thus increasing the security of the whole system.

Compression and encryption of signals add some delay, especially when powerful system for the Telemedicine unit PC is not used. This is the reason why both are added in the system as extra options, which can be disabled from Base unit user.

\section{Results - Discussion}

The final result is a "Multi-purpose" Telemedicine system, which facilitates a flexible architecture that can be adopted in several different application fields. The system has been tested and validated for a variety of medical devices and telecommunication means. Results presented in this section are typical for the needs of system use in Rural Health Centers, in Ambulance Vehicles or in a Navigating Ship.

Data transmission is done using the TCP/IP network protocol. Transmitting data over TCP/IP is a trivial and easy task when using networks, which have high bandwidth and low error rate. In order to transmit a buffer of $n$ bytes through TCP/IP a header of about 55 bytes is added, this will add a great amount of data especially in cases that we transmit small buffers (e.g. when transmitting a buffer of 10 bytes the network protocol will increase this buffer to 65 bytes). When transmitting a buffer that has size larger than the Maximum Transfer Unit (MTU) this buffer will be fragmented in to smaller packets that each one has the size of the MTU, all small packets will be reconnected when arriving at the destination site; this case will cause problems when one of the fragmentation packets is lost [42].

Considering the above two cases the transmission of data, especially through networks that have low bandwidth and high error rates (such as GSM mobile network and Satellite Links), has to be done in a way that will utilize the network use as much as possible. The buffers transmitted must have size that want be either too small or too big.

In order to measure the performance of TCP/IP over the GSM network several sizes of data buffers had been tested. The tests were performed using GSM modem, Nokia Card Phone 2.0 for the telemedicine unit, and a POTS modem US robotics sportster voice 56 KBPS for the base unit. These two devices support compression protocol V42 bis.

In order to perform the tests; buffers from 71 up to 479 bytes were selected; the size of buffers is proportional to

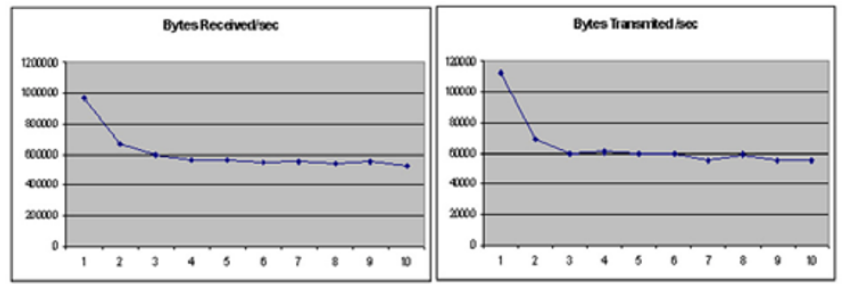

Figure 8

Received and Transmitted bytes per second, using several buffers

the data rate that the Propaq 2xx sends through the RS232 serial port. The packets had sizes: 71, 95, 143, 239, 287, $335,383,431,455,479$ bytes.

Using all the above buffers we made some measurements on the bytes that were received and transmitted to and from the base unit of the telemedicine system. Figure 8 shows the results of the bytes transmitted and received from the server unit when having a telemedicine unit connected with GSM to the server. Numbers 1 to 10 represent the size of the buffers used, 1 for the smallest ( 71 bytes) up to 10 for the largest ( 479 bytes). The mean value of the bytes transmitted/received per second was recorded for 2 minutes per case. As can be seen transmitting small packets of data cased the transmition of more bytes because of the overhead added on each buffer. The continuous transmition of small buffers also cased some problems on the communication and on the overall telemedicine unit operation; it could stop the operation of the protocol or add some problem when reading data from the medical monitor (too many system resources were used).

Having in mind all the above and the measurements of bytes transmitted (Figure 8 ) we had to select a buffer size that: would not add too much overhead to the transmitted data, would not cause fragmentation of the transmitted buffers and would not add too much delay on real time transmitted signal. Having in mind all the above the selected buffer size used was 431 bytes.

\section{Data transmition through GSM}

In order to measure the performance of the GSM network [43]-[45] using the optimum buffer size and the equipment mentioned above several measurements from a moving vehicle were performed. Measurements were performed using a moving vehicle in some of the main streets of the city of Athens (Greece). The vehicle was moving with a speed of approximately $60 \mathrm{~km} / \mathrm{h}$. The tests were performed in such a way and using the relevant equipment in order to simulate the data calls from an 
Table 3: Images transfer times - GSM and Inmarsat M satellite

\begin{tabular}{lllllll}
\hline File size & \multicolumn{2}{c}{ GSM } & & \multicolumn{2}{c}{ Inmarsat M satellite } \\
\cline { 2 - 3 } & Mean transfer time (100 files) $(\mathrm{sec})$ & Transfer rate (bps) & & Mean transfer time (100 files) (sec) & Transfer rate (bps) \\
\hline $6 \mathrm{~Kb}$ & 18 & 2666,7 & 40 & 1200 \\
$7 \mathrm{~Kb}$ & 20,5 & 2731,7 & 43 & 1302,3 \\
$8 \mathrm{~Kb}$ & 24 & 2666.7 & 46,5 & 1376,3 \\
$9 \mathrm{~Kb}$ & 26 & 2769,7 & 47 & 1531,9 \\
\hline
\end{tabular}

Table 4: Interruptions for GSM connections and Inmarsat M sattelite

\begin{tabular}{lll}
\hline Number of interruptions & GSM & Inmarsat M \\
\cline { 2 - 3 } & $\begin{array}{l}\text { Percentage from the total number of } \\
\text { interruptions }\end{array}$ & $\begin{array}{l}\text { Percentage from the total number of } \\
\text { interruptions }\end{array}$ \\
\hline 1 & $33,3 \%$ & $60 \%$ \\
2 & $16,7 \%$ & $20 \%$ \\
3 & $16,7 \%$ & $10 \%$ \\
4 & $25 \%$ & $10 \%$ \\
More & $8,3 \%$ & $0 \%$ \\
\hline
\end{tabular}

Ambulance vehicle. The transmission of ECG signals was done using continuous mode, while at the same time images of several sizes were transmitted. Tests were performed for one month using different routes and for different periods of a day.

Having performed 40 tests of approximately 30 minutes we had the following results:

a) In order to establish the connection between the telemedicine unit and the base unit an average time of $28 \mathrm{sec}-$ onds was required.

b) 10 images per test were successfully transmitted. The average transmition time for several image files was from 18 to 26 seconds (Table 3 ). Around 93\% of image transmissions were achieved within the first attempt; the rest $7 \%$ was transmitted using a second attempt because we had a line failure

c) The transmition of one ECG lead waveforms was performed in real time. The connection was interrupted once for at least $15 \%$ of all cases. In some cases we had more than one interruption (Table 4); reconnection of telemedicine unit to the base unit was performed successfully in all cases of interruption.

\section{Data transmition through Inmarsat satellite links}

In order to measure the performance of the system over the Inmarsat satellite network [46], several measurements were performed. Measurements were performed on a yacht using a mini $\mathrm{m}$ terminal for ships "Thrane \& Thrane TT-3064A CAPSAT Inmarsat Maritime Phone" for the telemedicine unit, and a POTS modem US robotics sportster voice 33.6 KBPS for the base unit. The mini $\mathrm{m}$ device is able to transmit data with a rate up to $2400 \mathrm{bps}$. The GSM optimum buffer size was used in this case too. The tests were performed in such a way and using the relevant equipment in order to simulate the emergency data calls from a yacht. The transmission of ECG signals was done using continuous mode, while at the same time images of several sizes were transmitted.

Having performed 40 tests of approximately 30 minutes for different periods of day, we had the following results:

a) In order to establish the connection between the telemedicine unit and the base unit an average time of $40 \mathrm{sec}-$ onds was required.

b) 10 images per tests were successfully transmitted. The average transmition time for several image files was from 40 to 47 seconds (Table 3 ). Around $90 \%$ of image transmissions were achieved within the first attempt; the rest $10 \%$ was transmitted using a second attempt because we had a line failure. 


\section{GREECE - Telemedicine network}

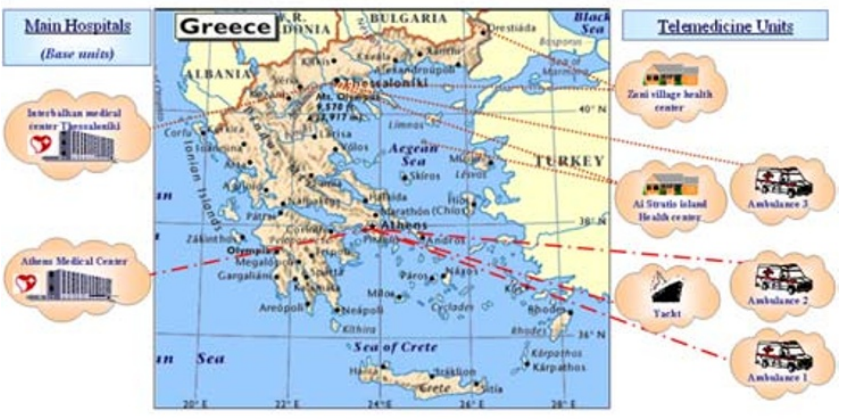

Figure 9

Telemedicine network - Greece

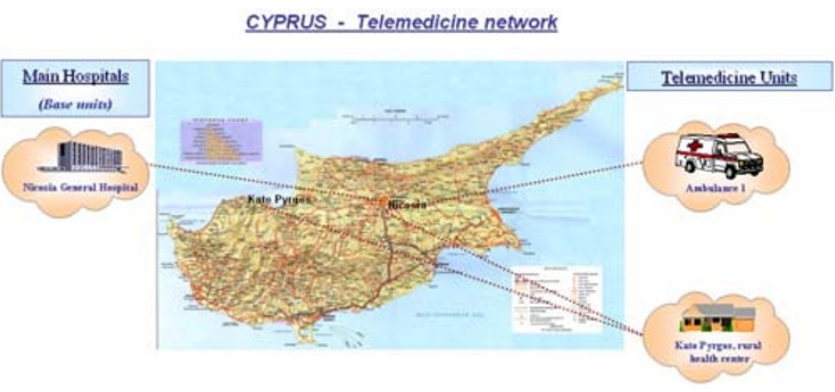

Figure 10

Telemedicine network - Cyprus

c) The transmition of two ECG lead waveforms and pulse oxymetry waveform was performed in real time. The connection was interrupted once for at least $20 \%$ of all cases, in some cases we had more than on interruptions (Table 4); reconnection of telemedicine unit to the base unit was performed successfully in all cases of interruption.

\section{Clinical Tests}

The system has been clinically tested through installation and extended validation of the system in a number of distinct demonstration sites across Europe.

More specifically, the use of the developed system in emergency cases handling in ambulances has been extensively demonstrated in Greece (Athens Medical Centre), Cyprus (Nicosia General Hospital), Italy (Azienda Ospedaliera Pisa) and Sweden (Malmo Ambulance Services). The initial demonstration of the system for ambulance emergency cases was performed on 100 (not severe) emergency cases for each hospital. The results of this phase were very promising. The system was able to improve, the percentage of incidents that in an emergency case initial diagnosis did not matched final diagnosis. For 100 cases without the system use, $13 \%$ of the initial diagnosis did not matched final diagnosis; while in 100 cases with the system use $8 \%$ of initial diagnosis did not matched the final diagnosis. The use of the system in Rural Health Centers has been tested extensively in Cyprus, where the national emergency system will be built on top of the already installed application. The use of the system in a Ship is currently being used in Athens Greece, and finally the use in home telecare is also being tested in Athens Greece. The system is currently installed and being used in two different countries, Greece (Figure 9) and Cyprus (Figure $10)$.

\section{Conclusions}

We have developed a medical device for telemedicine applications. The device uses GSM mobile telephony links, Satellite links or POTS links and allows the collection and transmission of vital biosignals, still images of the patient and bi-directional telepointing capability. The advance man-machine interface enhances the system functionality by allowing the users to operate in hands-free mode while receiving data and communicating with specialists. In order to introduce the system in daily health care provision; the system has been clinically tested using a controlled medical protocol. The final system is currently installed and used in two different countries Greece and Cyprus. Results from the system use are very promising thus encouraging us to continue the development and improvement of the system in order to be able to cover additional future needs.

\section{Acknowledgments}

The work described in this paper was initially based on two EU funded

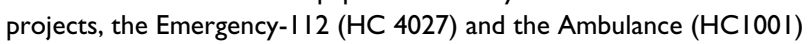
which were cost shared $R$ \& $D$ project funded by the European Commission under the Health Telematics Program. The work was extended and continued in order to be able to present the system described in this paper. Partners in the projects were ICCS-NTUA (project coordinator), Athens Medical Center Group, Medical Diagnosis and Treatment, Panafon and Epsilon Software from Greece, University of Cyprus and Nicosia Hospital in Cyprus, R \& S Informatica and CPR/Pisa Hospital from Italy, Eurotechnology and Malmo University Hospital in Sweden. We would like to express our thankfulness to all participants in the project for their significant contribution and fruitful collaboration.

\section{References}

I. Lin JC Applying Telecommunication Technology to Healthcare Delivery IEEE EMB Mag 1999, I8(4):28-3I

2. Guidelines For The Early Management Of Patient With Myocardial Infarction BM] 1994, 308:767-77|

3. Cerreli Ezio C 1997 Traffic Crashes Injuries And Fatalities, Preliminary Report National Highway Traffic Safety Administration, U.S. Dept of transportation 1997,

4. Hellenic National Statistics Service, Report 1997,

5. Evans T Cardiac Arrests Outside Hospital BM] 1998, 316:10311032 
6. David Sandler A Call to Needle Times After Acute Myocardial Infarction BMJ 1999, 3 18:1553

7. Canto JG, Rogers WJ and Bowlby LJ The Prehospital Electrocardiogram In Acute Myocardial Infarction: Is Its Full Potential Being Recognized? J Am College Cardiol 1997, 29:498-505

8. Stults KR, Brown DD, Schug VL and Bean JA Prehospital Defibrillation Performed By Emergency Medical Technicians In Rural Communities NEJM 1984, 3 1 0:219-223

9. Weaver WD, Copass MK, Hill DL, Fahrenbruch C, Hallstrom AP and Cobb LA Cardiac Arrest Treated With A New Automatic External Defibrillator By Out-Of-Hospital First Responders Am J Cardio 1986, 57:1017-1021

10. Cummins RO, Eisenberg MS, Litwin PE, Graves JR, Hearne TR and Hallstrom AP Automatic External Defibrillators Used By Emergency Medical Technicians: A Controlled Clinical Tria JAMA 1987, 257(1 2): 1605-1610

II. Schrading WA, Stein S, Eitel DR, Grove L, Horner L, Stechkert G, Sabulsky NK, Ogden CS and Hess DR. An Evaluation Of Automated Defibrillation And Manual Defibrillation By Emergency Medical Technicians In A Rural Setting Am J Emerg Med 1993 I I(2): I25-I 30

12. Shuster M and Keller JL. Effect Of Fire Department First-Responder Automated Defibrillation Annals Emerg Med 1993 22(4):72I-727

13. Sedgewick ML, Dalziel K, Watson J, Carrington DJ and Cobbe SM Performance Of An Established System Of First Responder Out-Of-Hospital Defibrillation. The Results Of The Second Year Of The Heartstart Scotland Project In The "Utstein Style" Resuscitation 1993, 26:75-88

14. Barro S, Presedo J, Castro D, Fernandez-Delgado M, Fraga S, Lama M and Vila J Intelligent Telemonitoring of Critical Care Patients IEEE EMB Mag 1999, 18(4):80-88

15. Strode S, Gustke S and Allen A Technical and Clinical Progress in Telemedicine Journal of American Medical Association 1999, 28I: 1066-1068

16. Wheeler T Strategies for delivering tele-home care-provider profiles Telemedicine Today 1998, 6:37-40

17. Deborah Dakins R Market Targets 1997 Telemedicine Magazine, Miller Freeman Inc June 1997

18. Bjorn Hofmann General Purpose Telemetry for Analog Biomedical Signals IEEE Engineering in Medicine and Biology Magazine 772-775Nov-Dec 1995

19. Murakami H, Shimizu K, Yamamoto K, Mikami T, Hishimiya N and Kondo K. Telemedicine Using Mobile Satellite Communication IEEE Trans on Biom Eng 1994, 4 I (5):488-497

20. Pavlopoulos S, Dembeyiotis S, Konnis G and Koutsouris D AMBU. LANCE - Mobile Unit for Health Care Provision via Telematics Support Proceedings of the IEEE Engineering in Medicine \& Biology, Amsterdam, The Netherlands 3430ctober 1996

21. Pavlopoulos S, Dembeyiotis S and Koutsouris D An Augmented Reality System for Health Care Provision via Telematics Support MMVR-97 Conference, San Diego, USA 1997,

22. Pavlopoulos S, Kyriakou E, Berler A, Dembeyiotis S and Koutsouris D A Novel Emergency Telemedicine System Based on Wireless Communication Technology - AMBULANCE IEEE Trans. Inform. Tech. Biomed. - Special Issue on Emerging Health Telematics Applications in Europe 1998, 2(4):261-267

23. Kyriacou Efthyvoulos, Pavlopoulos Sotiris, Dimitris Koutsouris, Andreas Andreou A, Pattichis Costas and Schizas Christos MULTIPURPOSE HEALTH CARE TELEMEDICINE SYSTEM Proceedings of the 23rd Annual International Conference of the IEEE/EMBS, Istanbul, Turkey 200I, 8. I:2-3

24. Bai J, Zhang Y, Shen D, Wen L, Ding C, Cui Z, Tian F, Yu B, Dai B and Dang J A Portable ECG and Blood Pressure Telemonitoring System IEEE EMB Mag 1999, 18(4):63-70

25. Morlion B, Verbandt $Y$, Paiva M, Estenne M, Michils A, Sandron P, Bawin C and Assis-Arantes P A Telemanagement System for Home Follow-up of Respiratory Patients IEEE EMB Mag 1999, | 8(4):7|-79

26. Pattichis CS, Kyriacou E Voskarides S, Pattichis MS, Istepanian R and Schizas CN Wireless Telemedicine Systems: An Overview IEEE Antennas \& Propagation Magazine 2002, 44(2): I 43- 153

27. Gagliano D Wireless Ambulance Telemedicine May Lessen Stroke Morbidity Telemedicine Today 1998, 6(1):21
28. Giovas P, Papadogiannis $D$ and Thomakos D Transmission Of Electrocardiograms From A Moving Ambulance Journal of Telemedicine and Telecare 1998, 4(1):5-7

29. Kontaxakis G, Walter S and Sakas G EU-TelelnViVo: An integrated portable telemedicine workstation featuring acquisition, processing and transmission over low-bandwidth lines of 3D ultrasound volume images Conf. Record, 2000 IEEE EMBS Intl Conf. Inf. Tech. Appl. Biomedicine (ITAB 2000), Arlington, VA, USA I58163November 2000

30. Sachpazidis I @Home: A modular telemedicine system Conf Record, 2nd Workshop Mobile Computing in Medicine, Heidelberg, Germany [http://www.mocomed.org/workshop2002/beitraege/Sachpazidis.pdf] April 2002

31. [http://telecom.esa.int/telecom/www/object/index.cfm?fobjectid=750]

32. Graschew G, Roelofs TA, Rakowsky S and Schlag PM Interactive telemedical applications in OP $\mathbf{2 0 0 0}$ via satellite Biomed Tech, Berlin, Germany 2002, 47(I):330-3

33. EMERGENCY I/2 project webpage [http://www.biomed.ntua.gr/ emergencyl I2]

34. Teixera S and Pacheco X Borland Delphi 5 Developers Guide, Sams Publishing, USA 1998,

35. Anagnostaki AP, Paylopoulos S, Kyriacou E and Koutsouris D A Novel Codification Scheme Based on the "VITAL" and "DICOM" Standards for Telemedicine IEEE Transactions on Biomedical Engineering, December 2002, 49( I 2):399- |4| I

36. DINAMAP PLUS monitor host communications, CRITIKON INC., 77659/B, USA 1994,

37. Propaq I xx communications option data interface guide, PROTOCOL SYSTEMS INC., Revision A, 810-0547-00, USA February 1994

38. Propaq Encore $2 x x$ External developers guide, PROTOCOL SYSTEMS INC. Revision A, 810-079/-00, USA July 1996

39. Pennebaker WB and Mitchell JL JPEG Still Image Data Compression Standard, Van-Nostrand - Reinhold 1993

40. Sayood K Introduction to Data Compression Morgan Kaufmann Publishers, Inc 1996, 27-54

4I. Schneier B Applied Cryptography John Wiley \& Sons 1996, 336-339

42. Stevens R TCPIIP Illustrated, Volume I. Addison-Wesley, USA I994,

43. Michel Mouly and Marie Pautet B The GSM System for Mobile Communications. France published by the authors 1992.

44. Jay Jayapalan and Mike Burke Cellular Data Services Architecture and Signalling IEEE Personal Communications, 2nd Quarter I994, 44-55

45. Balston DM and Moffett RH Data service handling in the GSM cellular radio system Electronic Engineering 79-84June 1990

46. Reinaldo Perez Wireless Communications Design Handbook, Volume I: Space (Interference: Aspects of Noise, Interference, and Environmental Concerns) Spacecraft Design Jet Propulsion Laboratory, California Institute of Technology Academic Press. San Diego, CA 1998

Publish with Biomed Central and every scientist can read your work free of charge

"BioMed Central will be the most significant development for disseminating the results of biomedical research in our lifetime. "

Sir Paul Nurse, Cancer Research UK

Your research papers will be:

- available free of charge to the entire biomedical community

- peer reviewed and published immediately upon acceptance

- cited in PubMed and archived on PubMed Centra

- yours - you keep the copyright 